\title{
Offenes Baukastensystem zur effizienten Dimensionierung von Materialflusssystemen
}

\author{
DR.-ING. INGOLF MEINHARDT \\ Prof. DR.-ING. HABIL. HANS-GEORG MARQUARDT \\ INSTITUT FÜR FÖRDERTECHNIK, BAUMASCHINEN UND LOGISTIK, TECHNISCHE UNIVERSITÄT DRESDEN
}

\section{Zusammenfassung}

Die optimale Gestaltung logistischer Systeme und Prozesse bekommt eine immer größere Bedeutung für die Wirtschaftlichkeit und Wettbewerbsfähigkeit von Unternehmen. Für Einzelkomponenten von Materialflusssystemen sind neben exakten analytischen Verfahren auch Näherungslösungen und Ersatzmodelle in Form von Polynomen, neuronalen Netzen oder zeitdiskreten Verfahren vorhanden, mit denen eine gute Nachbildung des Verhaltens dieser Komponenten möglich ist.

Ziel des Baukastensystems ist es, für diese Vielzahl von Methoden mit ihren spezifischen Ein- und Ausgangsgrößen eine übergeordnete, einheitliche Kommunikations- und Datenschnittstelle zu definieren. In einem grafischen Editor kann ein Modell eines Materialflusssystems aus solchen Bausteinen gebildet und parametriert werden. Durch Verbindungen zwischen den Bausteinen werden Informationen ausgetauscht. Die Berechnungen der Bausteine liefern Aussagen zu Auslastungen, Warteschlangen bzw. Wartezeiten vor den Bausteinen sowie Flussgrößen zur Beschreibung der Abgangströme.

\begin{abstract}
The optimal arrangement of logistical systems and operations gets an increased importance for the economicalness and competitiveness of enterprises. For individual components of material flow systems there are also existing approximate solutions and substitute models besides exact analytical calculations in the form of polynomials, neural nets or time-discrete analysis which allows a good analytical description of the behaviour of these components.

It is aim of the module system to define a superordinate and unified communication and data interface for all of these variety of methods with her specific input and output quantities. By using a graphic editor, the material flow system can be modelled of such components with specified functions and parameters. Connections between the components allows exchange of information. The calculations of the components provide statements concerning utilization, queue size or waiting time ahead of the components as well as parameters for the description of the departure process.
\end{abstract}

\section{Problemstellung}

Materialflusssysteme sind Träger innerbetrieblicher Transportprozesse und elementarer Bestandteil logistischer Systeme. Die optimale Gestaltung logistischer Systeme und Prozesse bekommt eine immer größere Bedeutung für die Wirtschaftlichkeit und Wettbewerbsfähigkeit von Unternehmen. Die effiziente Dimensionierung von Materialflusssystemen ist für Planer, Hersteller und Betreiber solcher Anlagen von grundsätzlicher Bedeutung. Für viele bei der Planung materialflusstechnischer Anlagen auftretende Fragestellungen steht noch immer kein Berechnungsverfahren oder -werkzeug zur Verfügung, welches allen drei folgenden Anforderungen gleichermaßen gerecht wird:

- $\quad$ Die Handhabung soll einfach, unkompliziert und schnell sein.

- Die Berechnungsergebnisse sollen eine hohe Genauigkeit haben.

- $\quad$ Die Berechnung soll allgemein gültige Ergebnisse liefern.

Dabei handelt es sich um Fragestellungen, die durchaus grundlegender Natur sind. Beispielsweise nach den (statistisch) zu erwartenden minimalen und maximalen Auftragsdurchlaufzeiten, nach dem Einfluss von Belastungsschwankungen auf die Anlagenleistung, nach vorzusehenden Puffern (Stauplätze) und Leistungsreserven (Auslastung). 


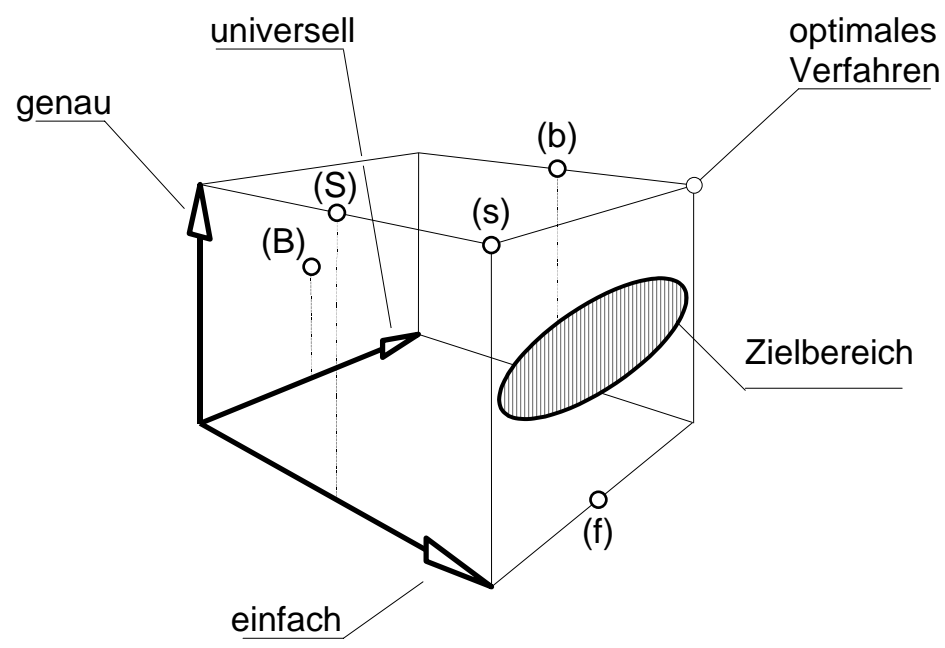

Abbildung 1: Anforderungen an Berechnungsmodelle [Schulze2000]

Für die oben genannten Aufgaben der Materialflussplanung stehen heute hauptsächlich drei Verfahren zur Verfügung (Abb. 1):

- Faustformeln (gekennzeichnet mit f) sind einfach aber ungenau. Das Systemverhalten von Materialflusskomponenten beschreiben sie selten über den gesamten Bereich möglicher Betriebsbedingungen und Konfigurationen. Das Verhalten von gesamten Materialflusssystemen ist zu komplex, als dass es mit Faustformeln adäquat beschreibbar wäre.

- Bedienungstheoretische Ansätze erlauben die Beschreibung von Materialflusskomponenten (kleines b) sehr genau und sehr umfassend, soweit Standardmethoden und -modelle der Bedienungstheorie anwendbar sind. Ist diese Voraussetzung nicht gegeben, kann der Aufwand zur Modellbildung schnell erheblich werden. Die Beschreibung von Materialflusssystemen (großes B) als Bedienungsnetzwerke ist nur unter (zum Teil stark) vereinfachenden Annahmen möglich. Solche Vereinfachungen gehen zu Lasten von Genauigkeit und Allgemeingültigkeit der Aussagen. Die Methoden sind häufig sehr komplex, ihre Anwendung erfordert vertiefte Kenntnisse in der Statistik und Stochastik.

- Simulationsuntersuchungen liefern für Materialflusskomponenten (kleines s) und für Materialflusssysteme (großes S) gleichermaßen genaue Aussagen. Der für die Untersuchungen erforderliche Aufwand hängt dabei weit weniger von den Eigenschaften und der Größe des Systems ab, als es bei bedienungstheoretischen Ansätzen der Fall ist. Die Aussagen der Simulation sind nie universell. Sie betreffen immer nur ein System in einer bestimmten Konfiguration. Die Anwendung der Simulation erfordert Spezialsoftware und vertiefte Kenntnisse in der Modellierung und Programmierung.

Verfahren, die genaue und allgemein gültige Aussagen über das Verhalten komplexer Materialflusssysteme liefern können, sind insbesondere in der Phase der Angebotserstellung bzw. in der Phase der Grobplanung von besonderer Wichtigkeit. Andererseits sind heute verfügbare Verfahren aber zu kompliziert und damit unwirtschaftlich. Gerade in der Phase der Systemgrobplanung werden häufig Änderungen in der Struktur des Systems notwendig, welche z.B. beim Einsatz der Simulation zu erheblichem Änderungsaufwand am Modell führt. Oftmals können solche Änderungen nicht schnell genug ausgeführt werden. Damit bleiben in der Praxis oft erhebliche Planungsunsicherheiten bestehen.

\section{Zielstellungen}

Der Grundgedanke des Baukastensystems besteht in der Modularisierung von Materialflusssystemen in einzelne Bausteine und Berechnungen zum Verhalten dieser Komponenten. Die betrachteten Module sind Materialflusskomponenten, die eine bestimmte logistische Funktion in einer konstruktiv bzw. steuerungstechnisch bedingten, definierten Weise ausführen. Das Verhalten einer Komponente wird durch Belastungen (Durchsatz) und technische Parameter (Geschwindigkeit, Schaltzeit o.ä.) beeinflusst und kann durch ein adäquates mathematisches Modell quantifiziert werden.

Das offene Baukastensystem soll dabei vor allem einen konzeptionellen Rahmen für die Integration derartiger Modellbausteine bilden. Es umfasst neben der Bausteinmodularisierung die Problematik der Kommunikation zwischen den Bausteinen (Schnittstellen) sowie Möglichkeiten zur Visualisierung von Ergebnissen.

Das daraus abgeleitete softwaretechnische Konzept berücksichtigt neben der einheitlichen Integration der zum Teil stark unterschiedlichen Berechnungsverfahren für einzelne Materialflusskomponenten auch einheitliche 
Definitionen zur Beschreibung von benötigten Eingangsparametern einschließlich der Randbedingungen (Definitionsbereich) und Plausibilitätskontrollen sowie zur Ergebnisbereitstellung. Äußerst wichtig war die Zielstellung, das System offen und erweiterbar zu gestalten: Prototypisch wurden zwar einzelne vorliegende Bausteine integriert, es ist aber jederzeit möglich, weitere Verfahren in Form eines Bausteines zu implementieren und in das Baukastensystem einzubringen.

Die Ergebnisse der Berechnungen für ein einzelnes Element (Output) fließen zugleich als Input in das nachfolgende Element ein: Genau wie im realen Materialflusssystem durch Aneinanderreihung einzelner fördertechnischer Elemente der Materialfluss realisiert wird, kommt es im Baukasten durch Verknüpfung der Bausteine zur Übertragung der relevanten Informationen, mit denen der Fluss beschrieben werden kann. Durch die Weitergabe der Ergebnisse kann trotz Modularisierung in einzelne Bausteine das Verhalten eines gesamten Materialflusssystems bestimmt werden. Daher sind auch hier einheitliche Festlegungen zu Art und Umfang der Übergabeparameter zwischen den Bausteinen erforderlich.

\section{Elemente des Baukastensystems}

\subsection{Bausteine}

Unter einem Baustein soll ein Modell einer Materialflusskomponente verstanden werden, welches das Verhalten dieser Komponente beim Vorliegen bestimmter Belastungen beschreibt. Dieses Verhalten ist insbesondere gekennzeichnet

- durch Warteschlangen und Wartezeiten, die vor der Komponente entstehen,

- $\quad$ durch Auslastung (Besetztanteil) der Komponente selbst und

- durch die Verteilung des zeitlichen Abstand (Variabilität) des die Komponente verlassenden Stroms an Transporteinheiten.

Maßgeblich bestimmt wird dieses Verhalten durch Intensität und Variabilität des ankommenden Stroms an Transporteinheiten, durch die Arbeitsweise (z.B. stetig / unstetig, stochastisch / deterministisch) und zeitliche Inanspruchnahme der Komponente sowie durch Steuerungsregeln, mit denen die Reihenfolge (Priorisierung / Vorfahrt) und/oder Dauer der Abarbeitung (z.B. Regalbediengerät mit Strategie „Minimierung des Leerfahrtanteils") verändert werden.

Im Grunde genommen beinhaltet ein Baustein damit ein mathematisches Modell, das einen oder mehrere ankommende Ströme von Transporteinheiten in einen oder mehrere abgehende Ströme transformiert (Abb. 2). Derartige Modelle gibt es beispielsweise in Form von

- $\quad$ Bedienmodellen ([Gnedenko1984], [Fischer1990 u.a.]),

- $\quad$ zeitdiskreten Modellen ([Arnold2005], [Furmans1992]),

- $\quad$ künstlichen neuronalen Netzen ([Schulze2000], [Markwardt2003]),

- $\quad$ Polynomen ([Schulze1998]).

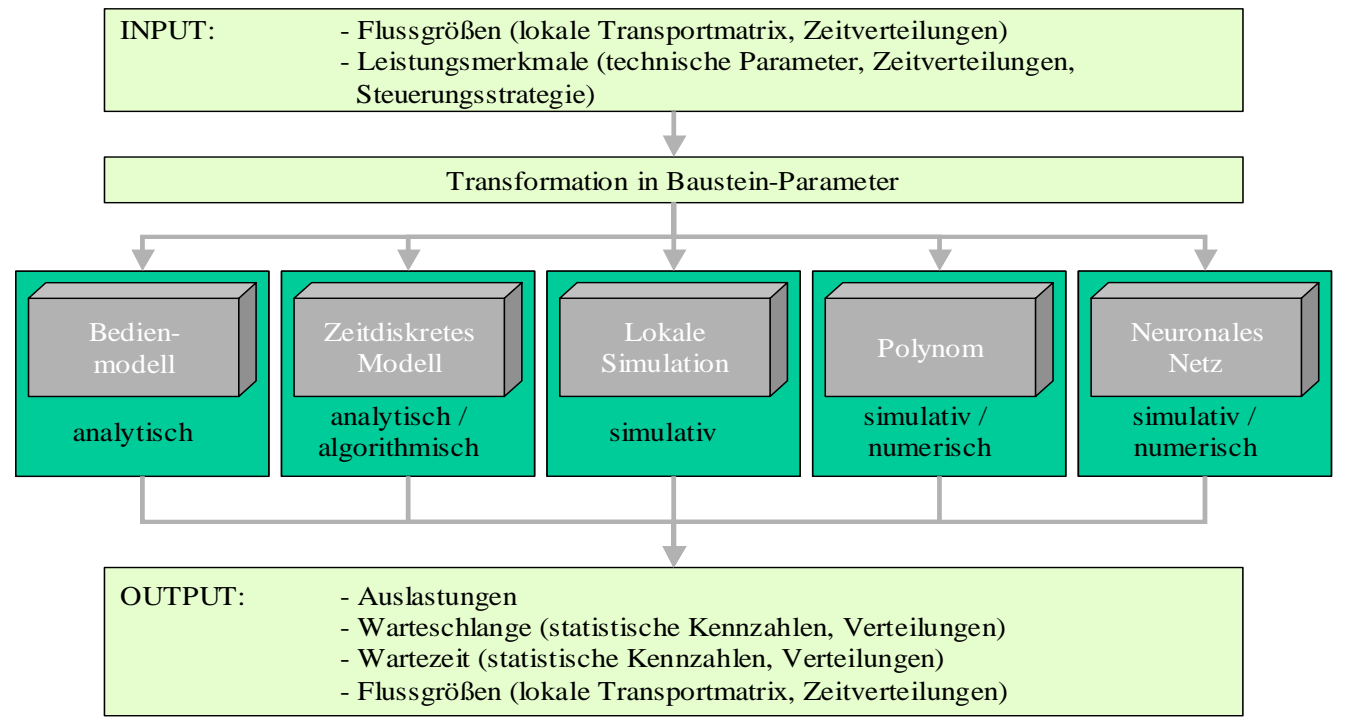

Abbildung 2: Anforderungen an Bausteine 
Die zu Grunde liegenden Verfahren (analytisch, simulativ, numerisch) unterscheiden sich zwar erheblich, genügen aber prinzipiell den genannten Anforderungen.

Die Fixierung auf ein mathematisches Modell ist aber nicht hinreichend, vielmehr bedarf es für einen Baustein auch definierter Schnittstellen, mit denen der Informationsaustausch erfolgen kann (Abb. 3). Dazu zählen neben der einheitlichen Bereitstellung von Informationen über die ankommenden und abgehenden Materialströme auch die Berücksichtigung einer individuellen Parametrierung der Bausteine sowie die Möglichkeit zur Interaktion mit dem Bediener (Anordnung, Parametrierung und Visualisierung).

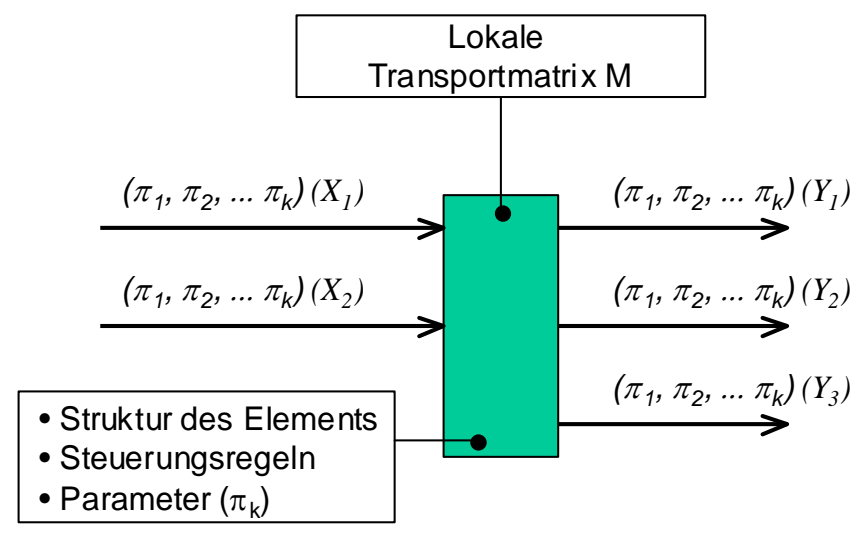

Abbildung 3: Formale Darstellung eines Bausteins und Schnittstellen [Markwardt2003]

Das offene Konzept erlaubt das eigenständige Entwickeln und Aufnehmen neuer Bausteine in den Baukasten. Dazu ergibt sich als weitere Anforderung die einfache Konfigurierbarkeit eines Bausteins hinsichtlich Identifikation, Aussehen und Leistungsbeschreibung. An einen Baustein innerhalb des Baukastensystems werden weiterhin die folgenden Anforderungen gestellt:

- Jeder Baustein ist eine in sich abgeschlossene Einheit und kann nur über die Ein- und Ausgänge mit seiner Umgebung kommunizieren. Damit ist ausgeschlossen, dass ein Baustein den Zustand eines anderen Bausteins beeinflussen kann. Das führt zu den beiden Lokalitätsbedingungen:

o Es gibt keine übergeordnete Steuerung, die in Abhängigkeit vom aktuellen Systemzustand dispositive Entscheidungen (z.B. zur Routenplanung) trifft.

o Blockierungen in Folge von Warteschlangen haben keine Auswirkungen auf die Funktion anderer Bausteine.

- Bausteine beinhalten in sich abgeschlossene Verfahren zur Dimensionierung einer Komponente (Klasse) des Materialflusssystems (z.B. Einschleusung auf einen Sorter, Drehtisch als Verzweigungselement oder als Eckumsetzer). Dabei werden auf Grund von technischen Parametern, Steuerungsstrategien und Belastungsannahmen (Durchsatz, Zeitverteilungen) Ergebnisse ermittelt.

- Ergebnisse im Sinne dieses Bausteinkonzepts sind Auslastungen, Warteschlangen bzw. Wartezeiten vor dem Baustein sowie Flussgrößen zur Beschreibung des Abgangstroms. Als Beschreibung eignen sich sowohl einzelne Kennwerte (Mittelwert, Varianz, Quantile) als auch statische Verteilungsfunktionen.

Die Lokalitätsbedingungen stellen Einschränkungen in der Anwendbarkeit des Baukastensystems dar: Systeme mit übergeordneten Steuerungsebenen wie Routenplanung oder Leerfahrzeugsteuerung, die Entscheidungen auf Grund der vorhandenen Transportaufträge und des aktuellen Systemzustands treffen (Fahrerlose Transportsysteme, Elektrohängebahn), können mit dem Baukasten nicht bearbeitet werden. Diese auf Unstetigförderern basierenden Systeme unterscheiden sich aber auch in ihren Einsatzmerkmalen grundlegend von den hier betrachteten Stetigförderersystemen.

Das Problem der Blockierungen vorgelagerter Bereiche durch zu große Warteschlangen kann dagegen bereits mit dem Baukasten betrachtet und zumindest visualisiert werden. Dazu ist den Verbindungen zwischen den Bausteinen eine Kapazität zugeordnet, so dass durch Vergleich mit den berechneten Warteschlangenlängen eine generelle Einschätzung zur Blockierungsgefahr möglich wird:

- Ist die Streckenkapazität kleiner als die mittlere Warteschlange, muss von einer permanenten Blockierung ausgegangen werden. In diesem Fall kann der vorhergehende Baustein seine gerade in Bearbeitung befindliche Transporteinheit nach dem Ende der „Bedienung“ nicht sofort abgeben und behindert damit auch seine weiteren ankommenden Transporteinheiten. Für die Transporteinheiten bedeutet das eine Verlustzeit, die auch nicht wieder aufgeholt werden kann, für das gesamte Transportsystem ist von einer Leistungsminderung (geringerer Durchsatz, größere Transport- / Durchlaufzeit) auszugehen. 
Da bei der Berechnung der Bausteine von einer Blockierfreiheit ausgegangen wird, sind die Berechnungsergebnisse in aller Regel falsch.

- $\quad$ Ist die Streckenkapazität zwar größer als die mittlere Warteschlange, aber kleiner als beispielsweise das 90\%-Quantil der Warteschlange, ist mit teilweisen Blockierungen (in dem Fall mit mehr als 10\% Wahrscheinlichkeit) zu rechnen. Dann tritt der o.g. Effekt nur zeitweise auf. Die Ergebnisse der Berechungen sind dann zumindest für einzelne Bausteine ungenau.

In beiden Fällen wird das Problem erkannt und dem Anwender signalisiert. Es wird davon ausgegangen, dass die geplante Funktionalität und Leistungsfähigkeit des Materialflusssystems nur dann gewährleistet ist, wenn keine Blockierungen auftreten. Durch Änderung der Parameter des kritischen Bausteins, aber auch durch Änderung der Materialströme muss daher eine Anpassung vorgenommen werden. Erst bei Vorliegen der Blockierfreiheit ist die Voraussetzung der Lokalität der Berechnungen erfüllt.

Die Berechnungsverfahren in den Bausteinen selbst können wegen der Modularisierung (Lokalität) sehr unterschiedlicher Art sein. Dabei ist es prinzipiell möglich, die einzelnen Ergebnisse eines Bausteins mit verschiedenen Verfahren zu ermitteln, insbesondere dann, wenn auf Grund eines eingeschränkten Definitionsbereichs der Eingangsparameter die Anwendung eines bestimmten Verfahrens nicht zulässig ist.

Bausteine, die einen Materialfluss auf Grund äußerer, nicht aus dem Verhalten des Bausteins resultierende Einflüsse generieren (Quelle) oder verändern (Service-Station), sind durch eine Flussgröße $\Phi$ parametriert. Die Flussgröße ist eine statistische Verteilungsfunktion zur Beschreibung der Ankunfts- und Abgangsströme (Zwischenankunftszeiten). In der Praxis, insbesondere in der Planungsphase, ist aber eine solche Verteilungsfunktion meist nicht bekannt. Zudem erweist sich das Rechnen mit Verteilungsfunktionen als numerisch aufwändig. Untersuchungen in [Markwardt2003] haben gezeigt, dass eine Parametrisierung als Abstraktion über statistische Verteilungsfunktionen mit gleichen Erwartungswerten, Minima und Streuungen ausreichend genaue Ergebnisse liefert.

Daher wird die Flussgröße beschrieben durch die Parameter Ankunftsrate $\lambda$ (=Durchsatz), Mindestzeitabstand $\mathrm{t}_{\mathrm{mind}}$ und Variationskoeffizient c (als Maß für die Variabilität des Stroms).

$$
\Phi=\mathrm{f}\left(\lambda, \mathrm{t}_{\text {mind }}, \mathrm{c}\right)
$$

Zur Visualisierung der Ergebnisse kann die dreiparametrige Gammaverteilung $f(t)=\Gamma(\alpha, \beta, \tau)$ zu Grunde gelegt werden, die eine gute Anpassung an reale Prozessverläufe bietet und durch die genannten Parameter eindeutig beschrieben ist:

$$
\begin{aligned}
& \alpha=\frac{\left(1-t_{\text {mind }} \cdot \lambda\right)^{2}}{c^{2}} \\
& \beta=\frac{c^{2}}{\lambda} \\
& \tau=t_{\text {mind }}
\end{aligned}
$$

Weitere leistungsbestimmende Größen wie technische Parameter, Zeitbedarfe u.ä. werden als Parametertupel $\pi(\mathrm{k})$ der jeweiligen Klasse zugeordnet.

So ist z.B. bei einer Einschleusung auf einen Sorter zu garantieren, dass der Strom auf der Hauptstrecke nicht angehalten wird. Das erfordert bei einer Einschleusung von der Nebenstrecke eine Lücke im Gutstrom auf der Hauptstrecke mit der Länge

$$
\mathrm{S}_{\text {mind, } \mathrm{E}}=\mathrm{S}_{\text {mind }}+\mathrm{v}_{\mathrm{F}} \cdot \mathrm{t}_{\mathrm{tr}}
$$

Mindestabstand und Fördergeschwindigkeit sind Parameter der ankommenden Förderstrecken, demnach ist lediglich die Größe $t_{t r}$ als Transferzeit ein leistungsbestimmender Parameter der Einschleusung.

\subsection{Förderstrecken}

Förderstrecken stellen die Verbindungen zwischen den Bausteinen her und realisieren den eigentlichen Materialfluss durch das System. Die technische Realisierung kann dabei prinzipiell durch verschiedenartige Bauformen von Stetig- und Unstetigförderern erfolgen. Systeme, die aber vollständig auf der Basis von Unstetigförderern arbeiten wie fahrerlose Transportsysteme (FTS) oder Elektrohängebahn (EHB), werden im Rahmen des Baukastens nicht betrachtet, weil die Lokalitätsbedingungen nicht gelten und beispielsweise eine übergeordnete Systemsteuerung (Fahrzeugdisposition, Leerfahrtoptimierung) einen erheblichen Einfluss auf die Leistungsfähigkeit des Gesamtsystems hat. 
Förderstrecken im hier verwendeten Sinne sind Rollen-, Ketten-, Bandförderer oder ähnliches, deren maximaler Durchsatz im Wesentlichen durch zwei Parameter bestimmt wird:

Fördergeschwindigkeit $\left(\mathrm{v}_{\mathrm{F}}\right)$ und Mindestabstand zwischen den Transporteinheiten $\left(\mathrm{s}_{\text {mind }}\right)$. Der Mindestabstand ergibt sich aus der Länge der Transporteinheit in Transportrichtung $\left(\mathrm{s}_{\mathrm{x}}\right)$ und einem Sicherheitsabstand $\left(\mathrm{s}_{0}\right)$, der

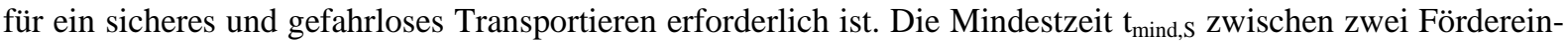
heiten auf einer Förderstrecke bestimmt sich demnach zu

$$
\mathrm{t}_{\text {mind, } \mathrm{S}}=\frac{\mathrm{s}_{\text {mind }}}{\mathrm{v}_{\mathrm{F}}}=\frac{\mathrm{s}_{\mathrm{x}}+\mathrm{s}_{0}}{\mathrm{v}_{\mathrm{F}}}
$$

Ist das verbindende Förderelement nicht staufähig (nicht akkumulierend, z.B. Gurtbandförderer), so kann sich der Abstand zwischen den Fördergütern während des Förder- oder Transportvorgangs nicht verändern: Muss das Band angehalten werden, weil eine Abgabe an das nachfolgende Förderelement nicht möglich ist, bleiben alle Einheiten stehen. In diesem Fall ist es also nicht möglich, die Lücken im Transportstrom zu schließen, die bereits bei der Aufgabe auf das Förderelement entstehen. Für die Berechnung der Mindestzeit $t_{\text {mind,s }}$ bedeutet das, dass dann auch die Mindestzeit $t_{\text {mind,B }}$ des vorhergehenden Bausteins berücksichtigt werden muss.

$$
\mathrm{t}_{\text {mind, } \mathrm{S}}=\max \left(\frac{\mathrm{s}_{\mathrm{x}}+\mathrm{s}_{0}}{\mathrm{v}_{\mathrm{F}}}, \mathrm{t}_{\text {mind, } \mathrm{B}}\right)
$$

Die Mindestzeit des Streckenelements nach (6) bzw. (7) wird als einer der Parameter der Flussgröße zur Beschreibung des am nachfolgenden Baustein ankommenden Stroms verwendet. Als Parameter der Förderstrecke werden neben der Fördergeschwindigkeit daher auch Angaben zum Transportgut (Abmessungen, Sicherheitsabstand, Transportrichtung) benötigt. Es bot sich ferner an, eine Typisierung der Förderstrecken hinsichtlich ihrer technischen Realisierung (Rollenförderer, Kettenförderer, Bandförderer usw. mit zugeordneten Parametern) vorzunehmen, um den Aufwand für die Beschreibung der Förderstrecken gering zu halten.

Weitere Parameter der Förderstrecken dienen der Aufnahme der Berechnungsergebnisse von vor- bzw. nachgelagerten Bausteinen und beinhalten:

- die Länge der Warteschlange (einzelne Kenngrößen wie Mittelwert, 90\%-, 95\% bzw. 99\%-Quantil oder - falls ermittelbar - als statistische Verteilung)

- $\quad$ die Wartezeit (ebenfalls Kenngrößen oder statistische Verteilung)

- die (Strecken-)Auslastung

- Variationskoeffizient für den Güterstrom

\subsection{Materialfluss}

Für die Darstellung des Materialflusses in einem System werden jeweils einzelne Materialfluss-Relationen betrachtet. Dabei wird angenommen, dass jede Relation

- $\quad$ an einer Quelle beginnt,

- $\quad$ an einer Senke endet,

- $\quad$ dabei mehrere Materialfluss-Komponenten (Bausteine) durchläuft und

- $\quad$ über den gesamten Verlauf in seiner Größe (Transportmenge) konstant bleibt.

Einziger leistungsbestimmender Parameter einer Materialfluss-Relation ist die Transportmenge. Sie wird als zeitabhängige Größe angegeben und entspricht damit dem Durchsatz. Mindestabstand und Variationskoeffizient werden vom erzeugenden Baustein (Quelle) bestimmt, von den weiteren durchlaufenen Bausteinen verändert und über die Förderstrecken jeweils an den nachfolgenden Baustein übertragen.

Die verbindenden Förderstrecken werden mit dem jeweiligen Durchsatz „belastet“. Bei Verbindungen, die von mehreren Relationen benutzt werden, summieren sich die Durchsätze, so dass sich unterschiedliche Streckenund Bausteinbelastungen ergeben.

\section{Metadaten zur Beschreibung der Elemente des Baukastens}

Im Kontext des Baukastensystems werden Metadaten ${ }^{1}$ verwendet, um die in einem Baustein enthaltenen Informationen über Anwendung, Verfahren und Restriktionen transparent zu machen. Ziel des Baukastensystems ist es je gerade, einfache und leicht handhabbare Berechnungsmodule für einen breiteren Anwenderkreis zur Verfügung zu stellen. Dazu sind Beschreibungen erforderlich, mit denen das Leistungsspektrum, mögliche Ergebnisse und Anwendungs- bzw. Einsatzkriterien dokumentiert werden.

\footnotetext{
${ }^{1}$ Unter Metadaten ("Daten über Daten") versteht man strukturierte Daten, mit deren Hilfe eine Informationsressource beschrieben und dadurch besser auffindbar gemacht wird. Der Begriff geht zwar dem Web-Zeitalter voraus, findet aber vor allem im Zusammenhang mit modernen elektronischen Informationssystemen seine Anwendung.
} 
Aufgabe der Baustein-Bibliothek ist die Sammlung, Verwaltung und Bereitstellung von Informationen über die vorhandenen Bausteine.

Damit soll dem Nutzer die Möglichkeit gegeben werden, für seine konkret benötigte Materialflusskomponente einen geeigneten Baustein zur Abbildung zu finden. Mit der Entwicklung weiterer Bausteine für ähnliche Funktionen, aber unterschiedliche Realisierungen (z. B. Regalbediengerät: einfach- oder doppeltiefe Lagerung, mit oder ohne Schnellläuferzone usw.) wächst die Notwendigkeit, die Einsatz- und Leistungsmerkmale des Bausteins in geeigneter Weise zu präsentieren.

Die Baustein-Bibliothek enthält demnach eine formalisierte Beschreibung der vorhandenen und verfügbaren Bausteine. Die Informationen sind im Wesentlichen unter dem Aspekt einer einheitlichen Identifikation, Information, Visualisierung und Implementierung der unterschiedlichen Bausteine zusammengestellt worden. Einige der in der Baustein-Bibliothek enthaltenen Metadaten lassen sich durchaus mehreren Rubriken zuordnen.

Identifikation und Information

Ein Baustein wird durch eine eindeutige Ident-Nummer fixiert. Daneben geben Informationen zum Autor (Entwicklung und/oder Implementierung des Verfahrens) und eine Funktionsbeschreibung eine verbale Auskunft über den Baustein. Zusätzlich ist jeder Baustein einem bestimmten Typ zugeordnet entsprechend der BausteinKlassifizierung (Bearbeiten, Verzweigen, Zusammenführen usw.), über den die Baustein-Auswahl eingegrenzt werden kann.

\section{Visualisierung}

Die Parameter für die Visualisierung beschreiben die Darstellung des Bausteins innerhalb des Baukastensystems (Form, Farbe, Lage der Ein- und Ausgänge des Bausteins, Icons).

Implementierung

Der Klassenname verweist auf die Implementierung des Bausteins. Zusätzlich benötigte Programm-Ressourcen (externe Bibliotheken wie *.dll ,*.tcl o.ä.) können angegeben werden. Weiterhin sind Bezeichnungen und Erläuterungen der erforderlichen technischen Parameter für den Eingabedialog enthalten.

Für die Förderstrecken wird ebenfalls eine formalisierte Beschreibung verwendet. Sie verweist jedoch nicht wie die Baustein-Bibliothek auf Software-Ressourcen, sondern enthält nur eine Reihe technischer Parameter, die für das Übertragungsverhalten der Förderstrecke eine Rolle spielen (Fördergeschwindigkeit, Arbeitsweise akkumulierend, Ausrichtung des Transportguts). Die Einträge lassen sich als Musterdatensätze (Template) für die Baustein-Verbindungen auffassen, um bestimmte, häufig vorkommende fördertechnische Lösungen diesen Verbindungen in einfacher Weise zuordnen zu können. Die Angaben sind aber im konkreten Anwendungsfall änderbar. Angaben zum Transportgut beschränken sich auf die Abmessungen der Transporteinheiten (Länge, Breite) und den erforderlichen Sicherheitsabstand $\left(\mathrm{s}_{0}\right)$. Als Grundform wird von einer Standard-Euro-Palette (1200x800 mm) ausgegangen, es lassen sich aber auch Güter mit anderen Maßen hinzufügen.

Die Angaben zum Transportgut werden in Verbindung mit den Parametern der Förderstrecken (Ausrichtung des Gutes längs oder quer) ausgewertet, so dass sich die jeweiligen Mindestabstände (Gleichung 6 bzw. 7) sowie der maximale Durchsatz $Q_{\max }$ als Grundlage für die Berechnung der Streckenauslastung bestimmen lassen.

\section{Umsetzung des Baukasten-Konzepts}

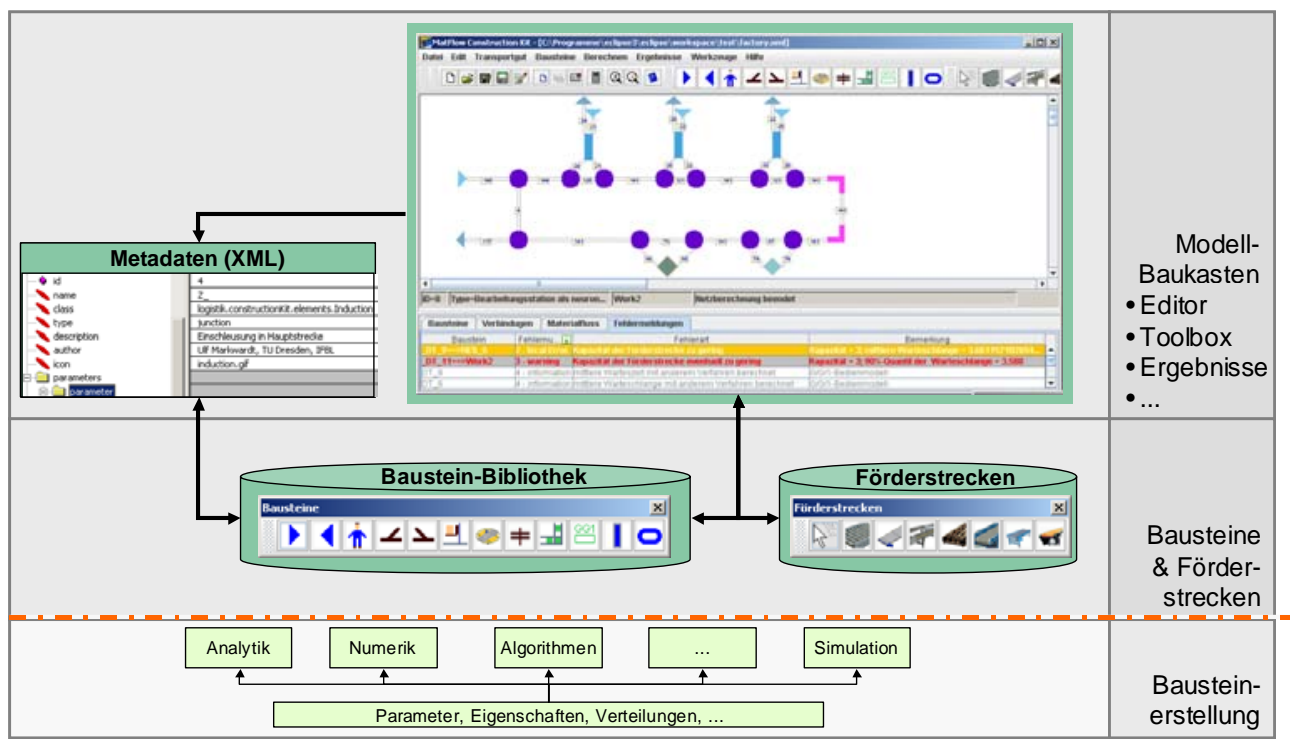




\section{Abbildung 4: Modell des Baukastensystems für die Materialflussdimensionierung}

Das Gesamtkonzept des Baukastensystems ist in Abbildung 4 dargestellt. Es besteht im Wesentlichen aus drei Bereichen:

- Bausteinerstellung

- Bausteinverwaltung (Bibliotheken)

- Baukasten (Benutzeroberfläche)

Dabei ist der Bereich der Bausteinerstellung nicht unmittelbarer Bestandteil der realisierten Lösung. Sie ist vielmehr die Quelle für die Bausteine, die über die jeweiligen Metadaten in einer Baustein-Bibliothek verwaltet und bereitgestellt werden.

Die Verwaltung von Bausteinen und Förderstrecken ist die Umsetzung der Baustein-Bibliothek und (im erweiterten Sinne) der Definitionen für die Förderstrecken.

Der Modellbaukasten selbst stellt die Grafische Nutzeroberfläche dar (Abb. 11) und enthält

- den interaktiven, grafischen Modelleditor,

- die Auswahlelemente (Werkzeugkoffer bzw. -filter) für Bausteine und Förderstrecken,

- $\quad$ tabellarische Übersichten für alle Bausteine, Förderstrecken und Materialflussrelationen sowie

- $\quad$ Eingabedialoge für Bausteine, Förderstrecken und Materialflussrelationen.

Die Entwicklung eines Modells mit dem Baukastensystem erfolgt prinzipiell in drei Schritten:

- Schritt eins umfasst die Anordnung und Definition der Bausteine. Der Modellbaukasten bietet die Möglichkeit, einen bestimmten Baustein direkt (z.B. Ausschleusung) oder unter Nutzung eines Bausteinfilters (z.B. alle Verzweigungselemente) auszuwählen und im grafischen Editor mittels Mausklick zu platzieren ${ }^{2}$. Anschließend erfolgt im Dialog die notwendige Parametrierung des Bausteins. Dies beinhaltet sowohl die Angaben zur Visualisierung (Drehung, Spiegelung) als auch die für die Dimensionierung erforderlichen technischen Parameter. Die für jeden Baustein benötigten Leistungsanforderungen (Durchsatz, lokale Transportmatrix) werden allerdings nicht direkt angegeben, sondern aus den Beziehungen zu den vor- und nachgelagerten Bausteinen automatisch ermittelt (Übertragungsfunktion der Förderstrecken).

- Danach erfolgt in einem zweiten Schritt die Definition von Verbindung zwischen den Bausteinen (Förderstrecken): Das Erzeugen der Bausteinverbindungen ist ebenfalls ganz einfach zu realisieren. Nach Auswahl der zu Grunde liegenden Fördertechnik (z.B. Rollenförderer) wird durch Ziehen des Mauszeigers von einem nicht belegten Ausgang zu einem nicht belegten Eingang eines Bausteins die entsprechende Förderstrecke erzeugt. In einem abschließenden Dialog können die gewählten Voreinstellungen zum Transportgut, zum Förderertyp usw. bestätigt oder gegebenenfalls korrigiert werden. Außerdem kann die Kapazität der Förderstrecke definiert werden. Dabei geht es weniger um die Länge des Förderers als viel mehr um die Anzahl der vorgesehenen Puffer- oder Stauplätze im Zusammenhang mit den zu berechnenden Warteschlangenlängen.

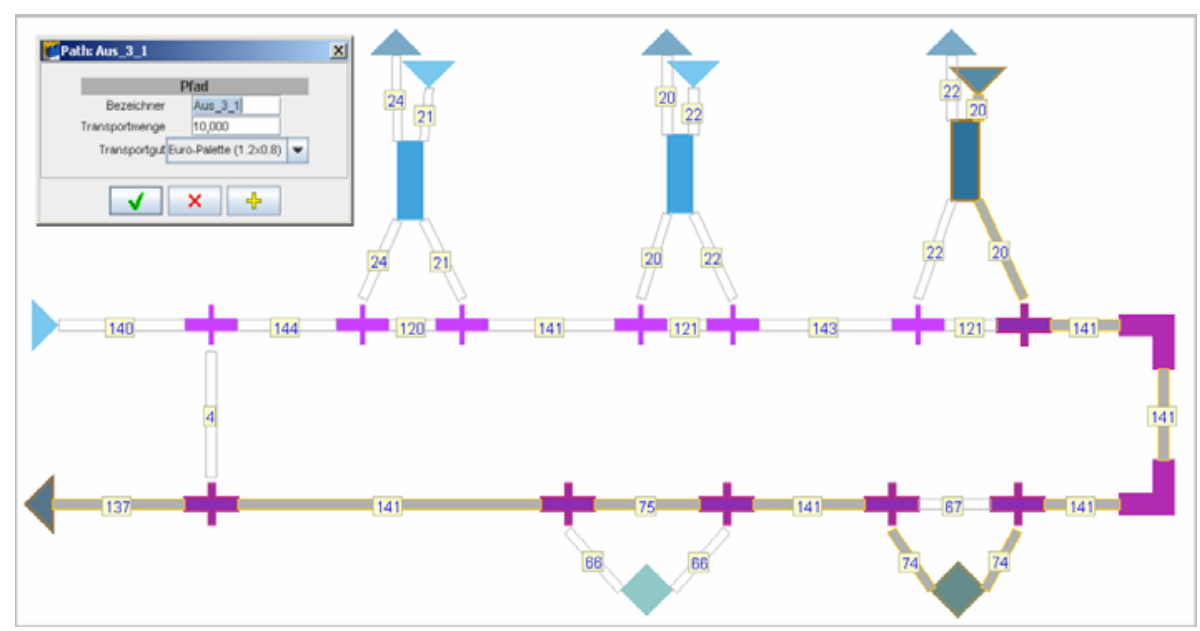

Abbildung 5: Definition eines Materialstroms von einer Quelle zu einer Senke

\footnotetext{
${ }^{2}$ Das zu erstellende Modell ist nicht maßstäblich, es ist im Allgemeinen ausreichend, die Anordnung der Bausteine nur der Struktur des Materialflusssystems entsprechend vorzunehmen.
} 
- Abschließend wird im dritten Schritt der Materialfluss definiert: Ein Materialstrom ist jeweils eine Relation, die an einer Quelle beginnt, an einer Senke endet und dabei mehrere Bausteine durchläuft. Da die Förderstrecken zu diesem Zeitpunkt bereits definiert sein müssen, kann automatisch ein möglicher Weg zwischen Quelle und Senke gefunden werden. Ähnlich wie bei Routenplanungssystemen kann dabei durch zusätzliche Angabe von Zwischenpunkten (via) der automatisch vorgeschlagene Transportweg verändert und angepasst werden (Abb. 5). Nach Bestätigung des Transportweges und damit der unterwegs zu passierenden Bausteine erfolgt in einem Dialog die Parametrierung (Transportmenge pro Stunde) für diese Relation. Die Elemente des Transportweges (die benutzten Förderstrecken) werden mit dem entsprechenden Durchsatz „belastet“.

Nach Abschluss der Modellierung kann die Berechnung ausgeführt werden. Im Ergebnis werden Kennzahlen bestimmt und im Baukasten in verschiedener Form visualisiert, um eine Bewertung der Ergebnisse vornehmen zu können.

Eine Übersicht Fehlermeldungen listet die Problemelemente auf. Dabei wird die Schwere eines Problems farblich hervorgehoben:

- fataler Fehler (rot): entsteht z.B. bei Überlastung eines Bausteins - die geforderte Leistung für einen Baustein (und damit die des Gesamtsystems) kann nicht erbracht werden.

- lokaler Fehler (orange): entsteht z.B. bei permanenter Blockierung - die mittlere Warteschlange vor einem Baustein ist größer als dessen vorgesehene Kapazität.

- Warnung (hellgelb): bei teilweiser Blockierung - das 90\%-Quantil der Warteschlange ist größer als die Kapazität der Förderstrecke, es ist daher zeitweise mit Blockierungen (und damit Behinderungen des vorhergehenden Bausteins) zu rechnen.

- Information (weiß): wird immer dann erzeugt, wenn Erwartungswerte für die Wartezeit oder Warteschlange mit einem G/G/1-Bedienmodell berechnet werden. Die Lösungen dieser Näherungsgleichungen sind im Allgemeinen nicht sehr genau, dienen aber als Abschätzung für die sonst fehlenden Kennwerte.

Entsprechend der berechneten Auslastung werden die Bausteine im Modelleditor mit einer Farbabstufung von Grün $(\rho<10 \%)$ nach Rot ( $\rho>90 \%)$ markiert, Bausteine und Förderstrecken leuchten rot bei Überlastung.

Die dargestellten Ergebnisse im Modelleditor zu Bausteinen und Förderstrecken sind umschaltbar durch den Nutzer (Abb. 6). Je nach den in den Bausteinen hinterlegten Berechnungen sind jedoch nicht immer alle Kenngrößen verfügbar.

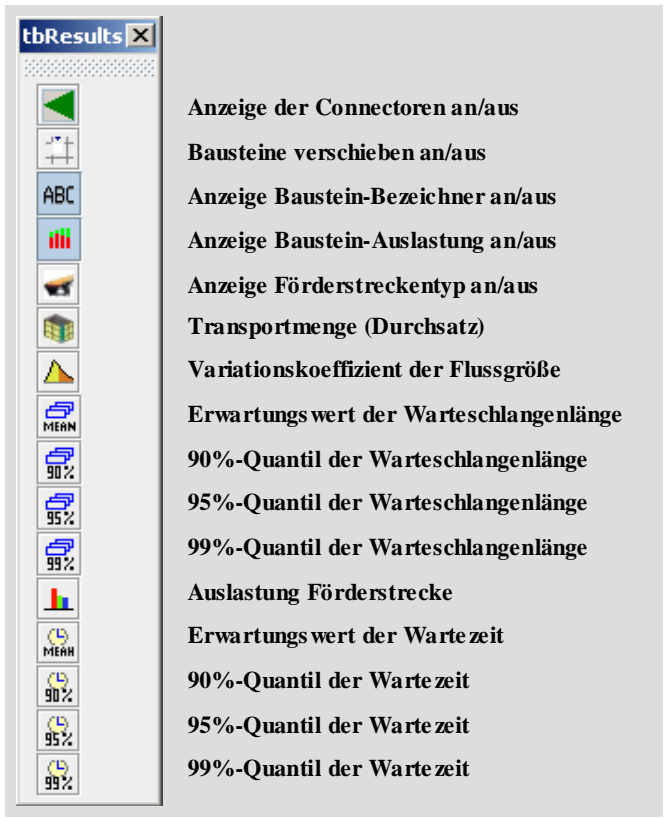

Abbildung 6: Umschaltbare Anzeige von Berechnungsergebnissen

\section{Erweiterbarkeit des Baukastens}

Die Implementierung des Baukastensystems wurde mit Java (Release 1.5) vorgenommen. Für das Kernsystem wird dabei das in Abbildung 7 dargestellte Klassen-Konzept umgesetzt. 
Ausgehend von einer allgemeinen Klasse (Object3D) für Visualisierung von und Interaktionen mit grafischen Objekten wurden für Bausteine (AbstractNode) und Förderstrecken (Connection) die jeweiligen Klassen abgeleitet. Für die Förderstecken ergibt sich dabei eine weitgehend einheitliche Beschreibungsform, die lediglich durch die Parametrierung (Vorlagen in der Förderstrecken-Bibliothek als XML-Datei) auf den konkreten Einsatz im Modell des Materialflusssystems angepasst werden muss.

Anders verhält es sich mit den Bausteinen: Durch die mögliche Vielfalt von Bausteinen und den ihnen zu Grunde liegenden Berechnungsverfahren muss es auch eine Vielzahl von Klassen geben. Um jedoch für jeden beliebigen Baustein den Zugriff (Bereitstellung von Eingangsdaten, Berechnung und Bereitstellung der Ergebnisse) in einer identischen Weise zu gewährleisten, muss es dafür eine nach außen einheitliche Schnittstelle geben.

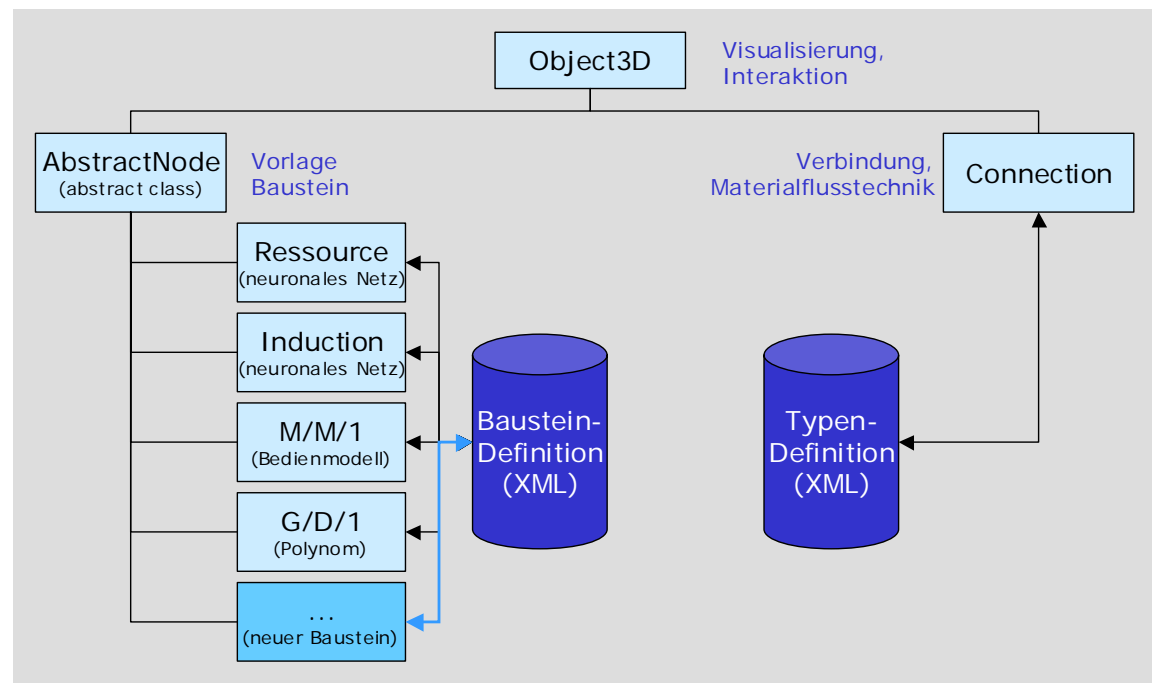

Abbildung 7: Klassen-Konzept für das Baukastensystem

Die Java zu Grunde liegende objektorientierte Programmierung bietet mit dem Konzept der „abstrakten Klasse“33 eine Möglichkeit, dies in einfacher Weise zu realisieren. Dazu wird mit AbstractNode quasi eine Vorlage entwickelt, von der alle implementierten Baustein-Klassen abgeleitet sind. AbstractNode selbst enthält alle Methoden, mit denen Baustein-Daten übernommen oder übergeben, die jeweiligen Visualisierungen vorgenommen, die baustein-internen Verbindungen (lokale Transportmatrix) verwaltet und Ein- und Ausgänge mit den zugehörigen Förderstrecken verbunden werden. Die für den Aufruf der eigentlichen Berechnungen in den Bausteinen verwendeten Methoden sind deklariert, aber nicht implementiert (sogenannte abstrakte Methoden).

Ein Baustein wird von AbstractNode abgeleitet und erbt damit die implementierten Methoden, lediglich die abstrakten Methoden, die die Spezifik des Bausteins ausmachen, sind noch zu implementieren.

Um neue Bausteine zu erzeugen, wird Unterstützung in Form eines Bildschirmdialogs angeboten (Abb. 8). Danach sind die entsprechenden Angaben zu den Metadaten, zur Struktur und zur Visualisierung des Bausteins, die Eingangsparameter (Name und Erläuterung) sowie die berechenbaren Ergebnisse (z.B. Auslastung, Quantile der Warteschlangenlänge, aber keine Aussage zu Wartezeiten usw.) anzugeben.

Nach Bestätigung der Daten und diversen Syntax- bzw. Semantik-Kontrollen wird der Baustein in der Bibliothek registriert, ein Sourcecode für den neuen Baustein generiert und kompiliert. Der Baustein selbst ist damit formal korrekt und kann sofort verwendet werden, liefert aber noch keine verwertbaren Ergebnisse, weil natürlich die Implementierung des Berechnungsverfahrens selbst noch aussteht. Das muss in einem zweiten Schritt im Rahmen der üblichen Software-Entwicklung nachgeholt werden. Dazu sind die Berechnungsverfahren zu implementieren und die Bausteinschnittstellen zu bedienen. Der generierte Java-Code enthält in den Kommentaren eine Reihe von Hinweisen für den Programmierer, so dass sich problemlos die Schnittstellen des Bausteins programmieren lassen (Abb. 9).

\footnotetext{
${ }^{3}$ Eine abstrakte Klasse ist eine Klasse, die selbst keine Instanzen besitzt. Sie definiert das gemeinsame, allgemeine Verhalten einer Gruppe von Objekten. Erst ihre Unterklassen, die weitere Spezialisierungen vornehmen, besitzen Instanzen.
} 


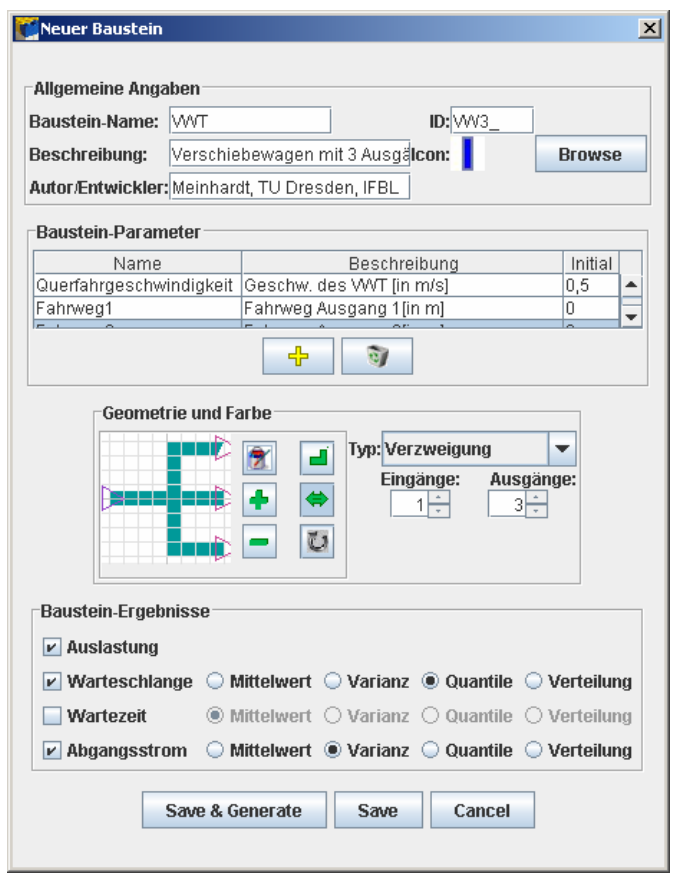

Abbildung 8: Dialog zur Erzeugung eines neuen Bausteins (Beispiel: VWT Verschiebewagen)

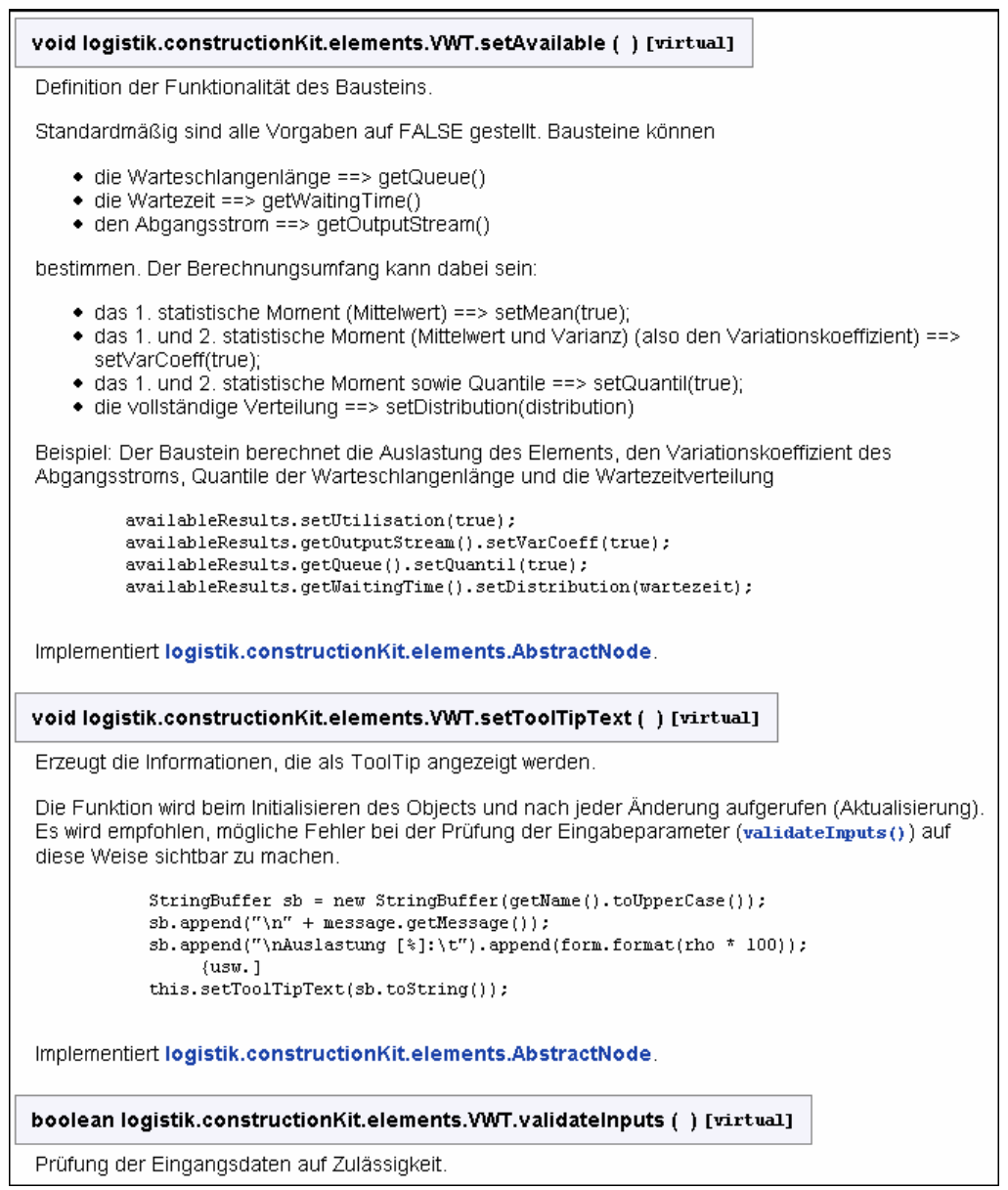

Abbildung 9: Mit JavaDoc generierte Dokumentation als Unterstützung für die Bausteinimplementierung 


\section{Anwendungsbeispiel}

In einem Beispiel werden ein Hochregallager (3 Regalbediengeräte) und zwei Kommissionierplätze durch ein Transportsystem verbunden. Mit der Einlastung von Kommissionieraufträgen werden im Simulationsmodell die entsprechenden Transportaufträge generiert und abgearbeitet (Abb. 10). Dabei können Systemzustände (z.B. Warteschlangen) protokolliert und statistisch ausgewertet werden.

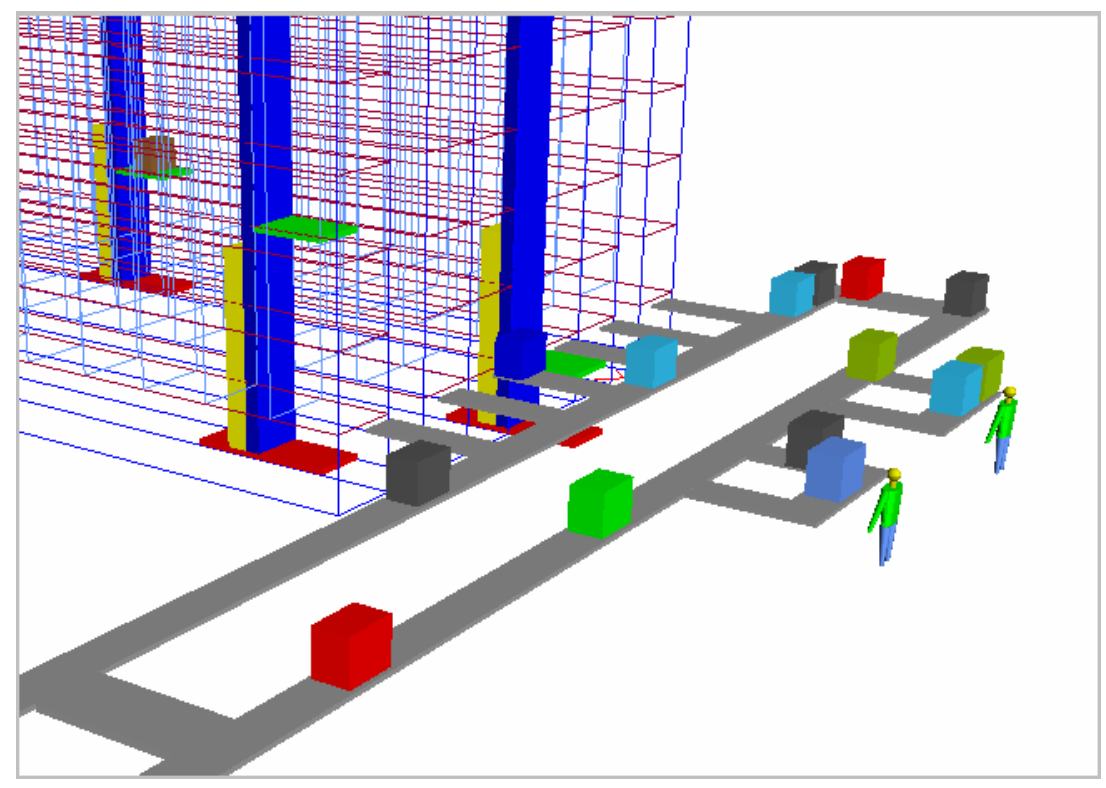

Abbildung 10: Lagervorzone und Kommissionierplätze im Simulationsmodell

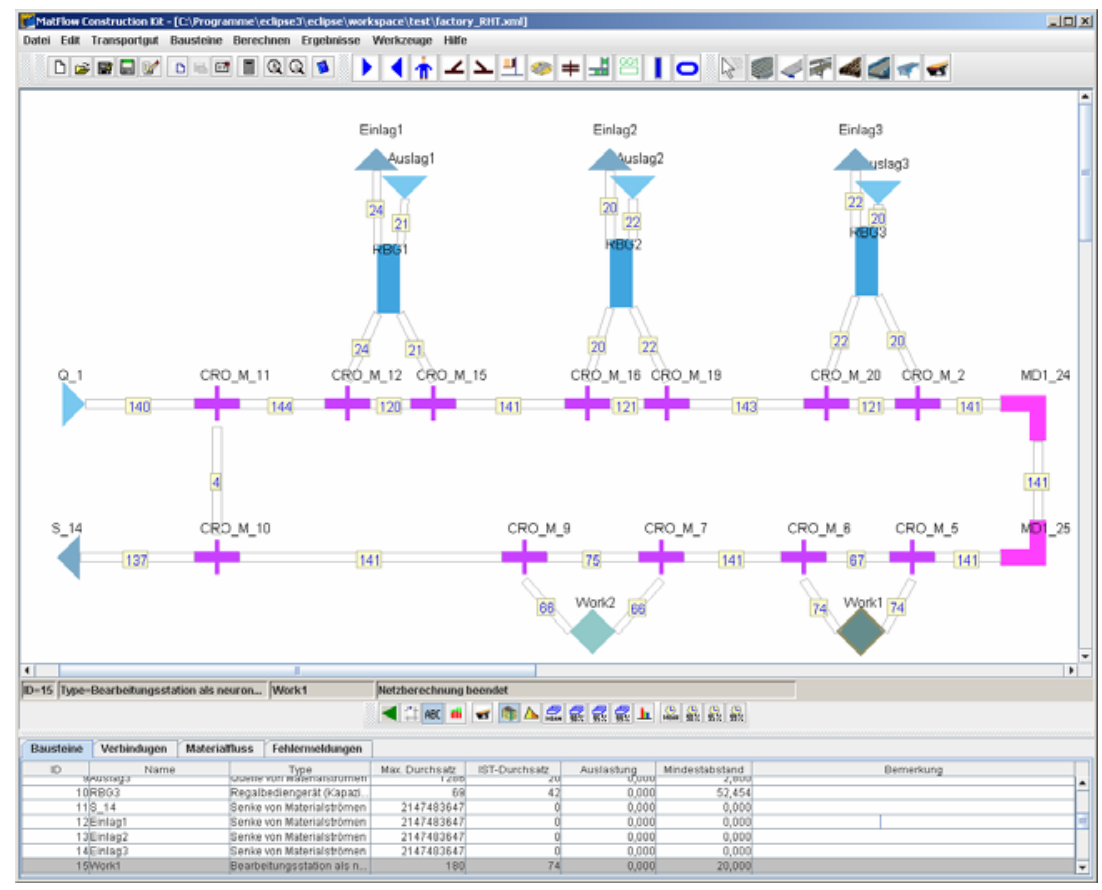

Abbildung 11: Lagervorzone und Kommissionierplätze im Baukasten

Ein entsprechendes Modell für den Baukasten ist in Abbildung 11 dargestellt.

Der Vorteil des Baukastensystems liegt selbst bei diesem recht einfachen Beispiel im Zeitvorteil: Für Erstellung und Test des Simulationsmodells und anschließende Simulationsläufe und Auswertungen wird ein Zeitaufwand 
von ca. 4-5 Stunden benötigt, das Baukastenmodell braucht für Erstellung und korrekte Parametrierung weniger als 0,5 Stunden, die Rechenzeit selbst ist vernachlässigbar gering.

Sollte im Ergebnis der Untersuchungen eine Änderung des Materialflusssystems notwendig werden, so führt das im Simulationsmodell teilweise zu erheblichen Änderungen (Abläufe, Steuerungsstrategien, Auswertungen) mit entsprechendem Zeitaufwand.

Im Baukasten können dagegen in einfacher Weise zusätzliche Bausteine eingefügt oder vorhandene ersetzt werden durch Bausteine mit geänderter Funktion oder Steuerung. Strukturelle Änderungen am Materialflusssystem sind also mit deutlich geringerem Aufwand realisierbar.

In [Markwardt2003] werden für mehrere Strukturen von Materialflusskomponenten Fehlerbetrachtungen über die Genauigkeit der mittels neuronaler Netze untersuchten Systeme gegenüber den Simulationsergebnissen vorgenommen. Danach ergibt sich beispielsweise für das 90\%-Quantil der Warteschlange eine Abweichung, die mit 90\% Sicherheit kleiner als 0,3 Warteplätze ist. Bei den Variationskoeffizienten des Abgangsstroms betragen die absoluten Abweichungen mit 90\% Sicherheit nicht mehr als 0,02 bis 0,05 (in Abhängigkeit vom betrachteten Baustein). Daraus wird die Schlussfolgerung abgeleitet, dass die durch Verknüpfung neuronaler Netze gewonnenen Aussagen sehr gut mit statistischen Ergebnissen diskreter Simulation übereinstimmen und eine Planungssicherheit ermöglichen, die für einen Grobentwurf von Materialflusssystemen weit über die heute gebräuchlichen statischen Berechnungsverfahren hinausgehen.

Im konkreten Beispiel wurde die Zahl der Pufferplätze vor den Kommissionierern (Work1 bzw. Work2) zunächst auf 3 begrenzt. Die Berechnung im Baukasten ergab dabei in beiden Fällen Fehlermeldungen mit dem Hinweis auf Blockierungen (Abb. 12, links). Diese bestätigten sich auch im Simulationsmodell (Abb. 12, rechts). Nach Vergrößerung der Pufferstrecken auf 7 Plätze ist die Blockierungsgefahr auf ein vertretbares Minimum reduziert, und die mit dem Baukasten berechneten Kenngrößen können durch die Simulation prinzipiell bestätigt werden (Tab. 1) ${ }^{4}$.

Tabelle. 1: Vergleich der Warteschlangen vor den Kommissionierplätzen

\begin{tabular}{|c|c|c|c|c|c|}
\hline & & \multicolumn{2}{|c|}{ Work1 } & \multicolumn{2}{|c|}{ Work2 } \\
\hline & & Simulation & Baukasten & Simulation & Baukasten \\
\hline Ankunftsrate & St/h & \multicolumn{2}{|c|}{74} & \multicolumn{2}{|c|}{66} \\
\hline mittlere Bedienzeit (Input) & [s] & \multicolumn{2}{|c|}{37,2} & \multicolumn{2}{|c|}{37,2} \\
\hline mittlere Wartezeit & [s] & 87,72 & - & 47,05 & - \\
\hline 90\%-Quantil der Wartezeit & [s] & 222,00 & - & 130,00 & - \\
\hline mittlere Warteschlange & Anzahl & 2,36 & 3,23 & 1,26 & 1,43 \\
\hline 90\%-Quantil der Warteschlange & Anzahl & 5,97 & 6,68 & 3,49 & 3,14 \\
\hline
\end{tabular}
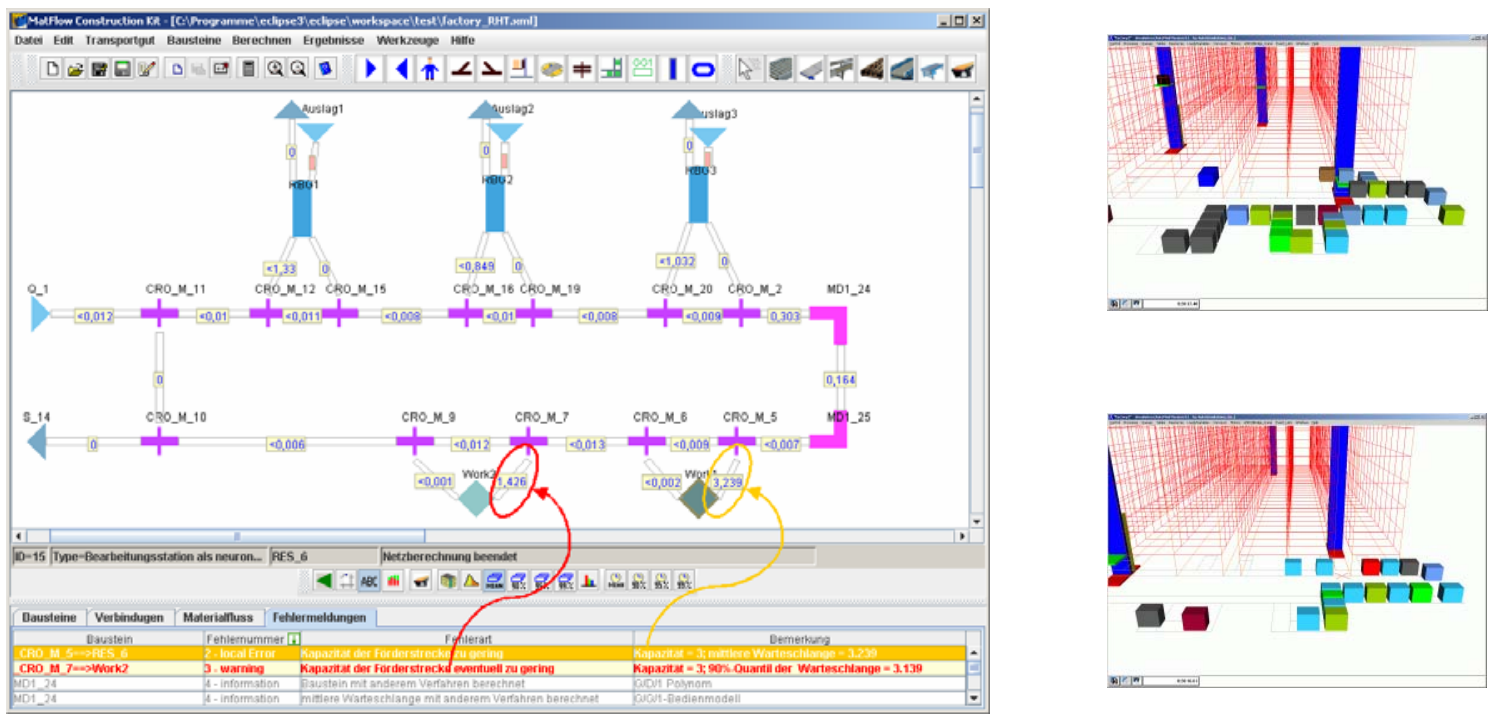

Abbildung 12: Visualisierung von zu kurzen Pufferstrecken im Baukasten (links) und im Simulationsmodell: Blockierungen führen zum Rückstau (rechts)

\footnotetext{
${ }^{4}$ Die Kommissionierer sind im Baukasten als Baustein Ressource abgebildet, für das die mittlere Warteschlange analytisch als G/G/1-Bedienmodell und das 90\%-Quantil mit einem neuronalen Netz berechnet wird.
} 


\section{Zusammenfassung}

Mit dem offenen Baukastensystem ist eine schnelle, einfache, sichere und damit wirtschaftlichere Dimensionierung von Materialflusssystemen möglich. Für den Anwender sind sofort statistisch abgesicherte und ausreichend genaue Ergebnisse ohne aufwändige Berechnungen verfügbar, womit sich die Planungsqualität erhöht. Besondere Anforderungen an Hard- und Software sind dabei nicht erforderlich.

Für die Dimensionierung der einzelnen Bausteine stehen Informationen aus der Bedienungstheorie, Simulationswissen und numerische Verfahren direkt und anwendungsbereit zur Verfügung. Es erlaubt eine deutlich vereinfachte Berechnung von statistischen Kenngrößen wie Quantile (statistische Obergrenzen) der Pufferbelegung, Auslastung von Einzelelementen und mittlere Auftragsdurchlaufzeit bei gleichzeitig erhöhter Genauigkeit. Ferner ist das Baukastensystem offen für eine Erweiterung um neue Bausteine, die neue oder spezielle fördertechnische Elemente abbilden oder zusätzliche Informationen liefern können.

Da auch komplexe Materialflusssysteme immer wieder aus einer begrenzten Anzahl unterschiedlicher Komponenten bestehen, können durch die Verknüpfung der Einzelbausteine auch Gesamtsysteme abgebildet werden. Die Verknüpfung der Bausteine über eine einheitliche Schnittstelle erlaubt Aussagen über das Verhalten der Gesamtanlage. Bei Einsatz des Baukastensystems sind in einer solchen Verknüpfung jederzeit Parameteränderungen möglich, deren Folgen sofort sichtbar werden. Die Zeit bis zum Vorliegen gesicherter, ausreichend genauer Ergebnisse wird dadurch drastisch verkürzt. Damit erwächst Variantenuntersuchungen bereits in frühen Planungsphasen neues Potential und kann zum entscheidenden Wettbewerbsvorteil werden.

\section{Literatur}

[Arnold2005]

[Fischer1990]

[Furmans1992]

[Gnedenko1984]

[Markwardt2003]

[Schulze1998]

[Schulze2000]

[Schulze2003]
Arnold, D., Furmans, K.: Materialfluss in Logistiksystemen. Berlin [u.a.]: Springer, 2005 Fischer, Klaus; Hertel, Günther: Bedienungsprozesse im Transportwesen: Grundlagen und Anwendungen der Bedienungstheorie. Berlin: transpress, 1990

Furmans, Kai: Ein Beitrag zur theoretischen Behandlung von Materialflusspuffern in Bediensystemnetzwerken. Karlsruhe, 1992 (Wissenschaftliche Berichte des Institutes für Fördertechnik der Universität Karlsruhe, 37)

Gnedenko, Boris; König, Dieter: Handbuch der Bedienungstheorie I/II. Berlin: AkademieVerlag, 1984

Markwardt, Ulf: Modellierung modularer Materialfluss-Systeme mit Hilfe von künstlichen neuronalen Netzen. Dresden: Technische Universität, Dissertation, 2003

Schulze, Frank; Marquardt, Hans-Georg: Systematische Untersuchung von MaterialflussStrukturen und ihre Beschreibung durch Polynom-Ansätze. In: Mertins, Kai (Hrsg.); Rabe, Markus (Hrsg.): Erfahrungen aus der Zukunft. Berlin: Fraunhofer-Institut für Produktionsanlagen und Konstruktionstechnik (IPK), 1998, S. 391-399.

Tagungsband 8. ASIM-Fachtagung Simulation in Produktion und Logistik Schulze, Frank: Neue Methoden zur Modellbildung für die Planung und Steuerung von Materialflusssystemen. Dresden: Technische Universität, Dissertation, 2000 Markwardt, Ulf; Schulze, Frank : Neuronale Netze als Meta-Modelle in der MaterialflussSimulation. In: Hohmann, Rüdiger (Hrsg.): Frontiers in Simulation. Delft: Society for Modeling and Simulation International, 2003, S. 397-402. - Tagungsband 17. Symposium Simulationstechnik ASIM 2003 